\title{
Short communication: Does early-life administration of a Megasphaera elsdenii probiotic affect long-term establishment of the organism in the rumen and alter rumen metabolism in the dairy calf?
}

\author{
T. T. Yohe, ${ }^{*}$ B. D. Enger, ${ }^{*}$ L. Wang, $\dagger$ H. L. M. Tucker, ${ }^{*}$ C. A. Ceh, ${ }^{*}$ C. L. M. Parsons, ${ }^{*} Z$. Yu, $\dagger$ and K. M. Daniels ${ }^{* 1}$ \\ *Dairy Science Department, Virginia Polytechnic Institute and State University, Blacksburg 24061 \\ †Animal Sciences Department, The Ohio State University, Columbus 43210
}

\begin{abstract}
Megasphaera elsdenii is a bacterial species of the rumen that can utilize lactate to produce butyrate, a key volatile fatty acid often implicated in driving calf rumen development. Because lactate is abundant in the rumen of young calves, administration of $M$. elsdenii to increase butyrate production and thus promote calf rumen development is an appealing possibility. The main objective of this study was to determine whether $M$. elsdenii administration to calves via oral drench at $14 \mathrm{~d}$ of age affected its long-term establishment at $70 \mathrm{~d}$ postadministration. Ruminal volatile fatty acid and lactate profiles and blood glucose and $\beta$-hydroxybutyrate concentrations were also examined to determine potential influence on rumen metabolism. Six neonatal Holstein heifer calves were blocked on $\mathrm{d} 1$ by body weight (41.3 $\pm 1.8 \mathrm{~kg})$ and total serum protein $(5.23 \pm 0.16 \mathrm{~g} / \mathrm{dL})$ and assigned to either the M. elsdenii $(\mathrm{n}=3)$ or control $(\mathrm{n}=3)$ treatment groups. On $\mathrm{d} 14$, calves in the $M$. elsdenii group orally received $25 \mathrm{~mL}$ of a commercially available $M$. elsdenii suspension, whereas calves in the control group received $25 \mathrm{~mL}$ of the same product that had been autoclaved. Rumen contents and blood samples were collected weekly from each animal until $84 \mathrm{~d}$ of age. The oral administration of $M$. elsdenii at $14 \mathrm{~d}$ did not increase the abundance of $M$. elsdenii $70 \mathrm{~d}$ postdosing, alter rumen fermentation, or change blood metabolites associated with butyrate. These results suggest that a single administration of the $M$. elsdenii probiotic may not affect the rumen establishment of the organism.
\end{abstract}

Key words: Lactipro, microbiome, rumen fermentation end product

Received January 4, 2017.

Accepted September 30, 2017.

${ }^{1}$ Corresponding author: danielsk@vt.edu

\section{Short Communication}

Rumen development is influenced by microbial fermentation of ingested feedstuffs to VFA, and butyrate is most often implicated in promoting rumen papillae development (Sander et al., 1959; Mentschel et al., 2001). One way to potentially increase ruminal butyrate production would be to enrich the rumen ecosystem with bacteria that produce butyrate as a fermentation end product. Megasphaera elsdenii is known to utilize lactate and produce butyrate (Elsden et al., 1956; Counotte et al., 1981), making it a bacterial species that might be used to increase rumen butyrate production. Lactate is not limiting in the rumen of very young dairy calves, and it primarily contributes to the low rumen $\mathrm{pH}(5.0-6.0)$ reported for periruminant calves (Gentile et al., 2004; Yohe et al., 2015).

Research with $M$. elsdenii and rumen function is not limited to calves and has a long history in adult ruminants (Hobson et al., 1958; Klieve et al., 2003; Henning et al., 2010; Aikman et al., 2011; Weimer et al., 2015). Klieve et al. (2003) examined ruminal M. elsdenii establishment in steers and found success when M. elsdenii was inoculated in combination with Butyrivibrio fibrisolvens. In contrast, Weimer et al. (2015) did not observe $M$. elsdenii establishment in lactating cows. It is clear that the efficacy of $M$. elsdenii probiotic varies. Recent research involving $M$. elsdenii administration to calves has shown promise as $M$. elsdenii-dosed calves had larger and a greater number of rumen papillae (Muya et al., 2015), improved feed efficiency pre- and postweaning (Muya et al., 2017), and increased plasma BHB concentrations (Muya et al., 2015, 2017). However, as noted earlier, demonstration that $M$. elsdenii probiotics establish in the rumen is generally lacking. We hypothesized that M. elsdenii could be used as a probiotic and effectively establish itself in the calf rumen. The main objective of this pilot study was to determine whether oral M. elsdenii administration to dairy calves at $14 \mathrm{~d}$ of age would help establish a persistent population of the organism after dosing. We also examined the effect 
on rumen VFA and lactate concentrations and blood glucose and BHB concentrations.

This experiment was approved by the Virginia Tech Animal Care and Use Committee (protocol no. 15-166) and conducted from September 2015 to January 2016. Six Holstein heifers born at Virginia Tech (Blacksburg) were used. Heifers were enrolled as they were born and were individually housed and fed in outdoor hutches on gravel with sawdust bedding. Hutch placement prevented calf-to-calf contact. Within $8 \mathrm{~h}$ of birth, each calf received $4 \mathrm{~L}$ of colostrum over 2 feedings. A single blood sample was obtained from each calf within $24 \mathrm{~h}$ of birth to determine total serum protein concentrations (Yohe et al., 2015).

On $\mathrm{d} 1$, calves were blocked by BW $(41.3 \pm 1.8$ $\mathrm{kg}$ ) and serum total protein taken within $24 \mathrm{~h}$ after colostrum administration $(5.23 \pm 0.16 \mathrm{~g} / \mathrm{dL})$ into 2 treatment groups: treatment $(\mathbf{P R O} ; \mathrm{n}=3)$ and control $(\mathbf{C O N} ; \mathrm{n}=3)$. On d 14, calves assigned to PRO received $25 \mathrm{~mL}$ of a commercially available M. elsdenii suspension (Lactipro; MS Biotec, Wamego, KS), and calves assigned to $\mathrm{CON}$ received $25 \mathrm{~mL}$ of the same product that had been autoclaved. Lactipro contains approximately $2 \times 10^{8} \mathrm{cfu} / \mathrm{mL}$ of $M$. elsdenii NCIMB 41125 according to the manufacturer. Lactipro was received directly from the manufacturer, stored at room temperature, and used within $14 \mathrm{~d}$ of receipt. Because M. elsdenii is a strict anaerobe, anaerobiosis of the product was visually confirmed (lack of blue color) immediately before administration.

All calves were fed the same nonmedicated milk replacer (MR; Amplifier Max; Land O'Lakes Animal Milk Products Co., Shoreview, MN) containing 22.7\% $\mathrm{CP}$ and $21.9 \%$ fat (DM basis). Milk replacer composition included milk protein sources and animal fat. The MR was reconstituted to $13 \%$ solids and fed by bottles until calves were bucket trained. Calves began a 5-d weaning process when $0.91 \mathrm{~kg}$ of starter was consumed for 3 consecutive days or at d 42 , whichever came first. Calves were fed MR at morning (0700 h) but not evening $(1900 \mathrm{~h}$ ) feedings during the weaning process. Pelleted starter (Southern States Cooperative, Richmond, VA) and water were available ad libitum to all calves from d 0 . Starter composition included approximately $26.3 \%$ high-protein soybean meal, $23.2 \%$ ground soybean hulls, $11.8 \%$ wheat middlings, $11.5 \%$ ground wheat, $11.5 \%$ ground barley, $7.0 \%$ cottonseed hulls, $3.4 \%$ ground corn, $1.8 \%$ ground limestone, and $1.2 \%$ molasses $(23.6 \% \mathrm{CP}, 31.4 \% \mathrm{NDF}$, and $17.0 \%$ starch on a DM basis). Weekly samples of MR and starter were combined, mixed, and sent to Cumberland Valley Analytical Lab (Hagerstown, MD) for analysis.

Heifer was the experimental unit. Feed intake and fecal and respiratory scores were recorded daily (Yohe et al., 2015) and did not differ by treatment (data not shown).

Rumen fluid samples (20-50 mL) were obtained weekly and at 15,16, and $18 \mathrm{~d}$ of age, resulting in 15 rumen samples per calf. Samples were collected via oresophageal tube at $0900 \mathrm{~h}$ using an attached $60-\mathrm{mL}$ syringe for aspiration; $\mathrm{pH}$ measures were taken at sampling. Approximately $10 \mathrm{~mL}$ of each rumen sample was immediately transferred into sterile $15-\mathrm{mL}$ polystyrene conical tubes and stored at $-20^{\circ} \mathrm{C}$ for rumen microbiome analysis. The remaining rumen fluid was strained through 4 layers of cheesecloth and stored at $-20^{\circ} \mathrm{C}$ in glass screw-top tubes for VFA and lactate analyses. The VFA was analyzed using GC using an adapted method from Kristensen (2000); D- and L-lactate were analyzed using an enzymatic test kit (Boehringer Mannheim D/L-Lactic Acid Test Combination; R-Biopharm AG, Darmstadt, Germany).

Rumen sample metagenomic DNA was extracted using the method of $\mathrm{Yu}$ and Morrison (2004) to determine Megasphaera prevalence and to analyze changes in rumen bacterial populations. Deoxyribonucleic acid was quantified on a NanoDrop (Thermo Fisher Scientific, Waltham, MA), diluted to $10 \mathrm{ng} /$ $\mu \mathrm{L}$, frozen at $-20^{\circ} \mathrm{C}$, and shipped overnight to the Molecular Cell Imaging Center at The Ohio State University (Wooster, $\mathrm{OH}$ ) for $16 \mathrm{~S}$ rRNA gene amplicon sequencing. Briefly, the V4-V5 hypervariable region of the 16S rRNA gene of each sample was amplified using universal primers $515 \mathrm{~F}$ and 806R, with each library having a unique barcode. Amplicon libraries were pooled at equal molar ratios and sequenced using the V3 kit $(2 \times 300$ paired-end kit $)$ on an Illumina (San Diego, CA) MiSeq platform. The sequencing data were analyzed using QIIME (Caporaso et al., 2010) as described by Kigerl et al. (2016). Bases with quality scores lower than 25 were removed, and then paired reads were joined together using the fastq-join algorithm (Aronesty, 2011). Clean sequences shorter than $250 \mathrm{bp}$ were removed. Chimera sequences were identified using ChimeraSlayer (Haas et al., 2011) and filtered out. Species-equivalent operational taxonomic units (OTU) were clustered using the pick open reference method against the Silva_119_release reference sequences at $97 \%$ similarity using the uclust algorithm of QIIME (Edgar, 2010). Taxonomic assignment of OTU was performed by comparing the representative sequence of each OTU against the same Silva reference database using the uclust consensus taxonomy assigner of QIIME (Edgar, 2010). Minor OTU each represented by either $<0.005 \%$ of total sequences or $<0.1 \%$ in all the samples were discarded (Bokulich et al., 2013). The sequences were deposited in the GenBank SRA database with the accession number SRP092199. 
Jugular blood $(5 \mathrm{~mL})$ was collected from each calf weekly, $2 \mathrm{~h}$ after the morning feeding $(0900 \mathrm{~h})$, to determine glucose and $\mathrm{BHB}$ concentrations. Coinciding with treatment administration, blood was also obtained on d 15, 16, and 18 at $0900 \mathrm{~h}$, yielding a total of 15 samples for each calf. Blood was collected into evacuated $\mathrm{NaF} / \mathrm{K}$ oxalate (glucose samples; $2.5 \mathrm{~mL}$ ) and $\mathrm{Na}$ heparin (BHB samples; $2.5 \mathrm{~mL}$ ) tubes. Plasma was harvested, stored at $-20^{\circ} \mathrm{C}$, and analyzed as previously described (Yohe et al., 2015).

Body weight gain, feed intake, blood metabolites, and rumen data (except for microbiome data) were analyzed using the MIXED procedure of SAS 9.3 (SAS Institute, Cary, NC). Calf BW, intake, blood glucose and $\mathrm{BHB}$, and ruminal VFA, $\mathrm{pH}$, and lactate models included the fixed effects of treatment, day, and their interaction. Day was specified rather than week to accommodate the additional blood and rumen samples collected around probiotic administration. Calf nested within treatment was a random effect in all models. All analyses included the best-fit covariance structure; denominator degrees of freedom were not specified. Orthogonal polynomial contrasts were performed when appropriate, and least squares means \pm standard errors of the mean are reported.

Microbiome data were compared among all the samples using principal coordinates analysis using the Bray-Curtis dissimilarity matrix that was calculated from 10,000 sequences rarefied from each sample. The relative abundance of individual OTU was calculated by dividing the number of sequences representing each OTU by the total number of sequences of respective samples. A $t$-test was performed to determine significant difference in relative abundance of OTU between the PRO and CON treatments.

The first objective of this trial was to determine whether Lactipro administration at $14 \mathrm{~d}$ of age would establish a persistent population of $M$. elsdenii in the calf rumen $70 \mathrm{~d}$ after dosing. As illustrated in Figure 1, the oral dosing only transiently increased the population of M. elsdenii. Accordingly, data collected concerning calf growth and MR and starter intakes did not differ between treatment groups and are not discussed in detail. Overall, mean final calf BW was $96.6 \pm 4.8$ $\mathrm{kg}$, and calves consumed mean totals of $20.8 \pm 0.7$ $\mathrm{kg}$ of MR and $114.5 \pm 14.5 \mathrm{~kg}$ of starter throughout the 84-d trial. Rumen and blood metabolites did not differ between treatment groups (Supplemental Table S1; https://doi.org/10.3168/jds.2017-12551) and were similar to values reported for periruminant calves (Quigley et al., 1994; Klotz and Heitmann, 2006). It should be noted that a single dose of Lactipro to calves did not alter ruminal butyrate concentration $(P=$ 0.38; Supplemental Table S1) and thus does not sup-

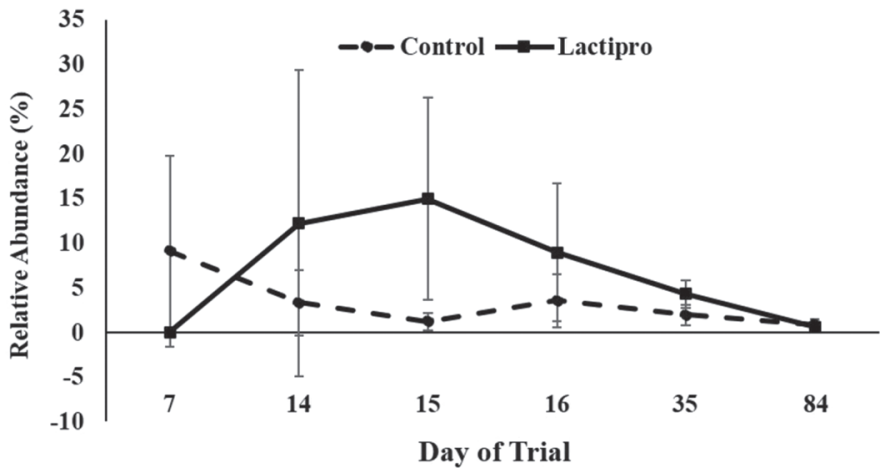

Figure 1. Mean relative abundance $( \pm$ SEM $)$ of the genus Megasphaera in the rumen of calves. Control $=$ calves received $25 \mathrm{~mL}$ of autoclaved Lactipro (MS Biotec, Wamego, KS); Lactipro = calves received $25 \mathrm{~mL}$ of nonautoclaved Lactipro, a commercially available probiotic product of Megasphaera elsdenii. Calves were dosed on d 14 of the trial.

port the narrative that Lactipro administration leads to increased ruminal butyrate production.

Administration of $M$. elsdenii had little effect on the overall rumen microbiome composition as illustrated by principal coordinates analysis (Figure 2), but the rumen microbiome composition showed temporal successions as the calves grew. Few OTU differed $7 \mathrm{~d}$ before the dosing, but more OTU appeared to differ in relative abundance between the Lactipro and control groups postdosing (Supplemental Table S2; https://doi.org/10 .3168/jds.2017-12551). Overall, several OTU assigned to the genus Bifidobacterium increased in relative abundance following Lactipro dosing, but the increase was no longer detected by d 35. Bifidobacteria are known fermenters of starch and simple sugars (Trovatelli and Matteuzzi, 1976), which would have been abundant in these calf rumens given the diet of easily fermentable feedstuffs. Bifidobacteria can also utilize other substrates, including lactose, fructo-oligosaccharides, and raffinose (Amaretti et al., 2006). The ruminal environment promoted by the introduction of $M$. elsdenii may have helped promote bifidobacteria growth. Within the genus Prevotella, the relative abundance of some OTU increased at d 2 postdosing, whereas some other OTU decreased in relative abundance 3 wk after dosing. Prevotella is one of the most abundant genera present in rumens of calves fed solid feed (Kim et al., 2016) and is known to utilize a wide variety of substrates (e.g., different forms of carbohydrates and proteins or peptides) to promote growth (Stevenson and Weimer, 2007). As with bifidobacteria, there may have been the creation of a beneficial rumen environment for growth of Prevotella after introduction of $M$. elsdenii that was lacking in the control group. Some OTU within the genus Veillonella also increased their relative abundance at 2 d after dosing. $\mathrm{Ng}$ and Hamilton (1971) and Mikx 
and van der Hoeven (1975) demonstrated the ability of Veillonella to utilize lactate, which, like M. elsdenii, would benefit from the highly fermentable diet resulting in increased lactate concentrations in the rumen (Supplemental Table S1). The difference between the control and probiotic-dosed calves suggests (just like other bacterial populations) a beneficial environment for Veillonella growth. The relative abundance of the genus Megasphaera numerically differed at d 7 before the dosing, but overall the Lactipro dosing only transiently increased the population of this genus, and by $\mathrm{d}$ 84 (70 d postdosing) no difference in relative abundance was noted (Figure 2). Collectively, Lactipro administration did not considerably alter the ruminal microbiome, and only a small number of bacterial genera were affected. Because the control group received autoclaved Lactipro, the dosing effect should be attributed to the activity of the Lactipro product.

Muya et al. (2015) recently reported that Lactipro administration at $14 \mathrm{~d}$ of age increased rumen butyrate concentrations relative to calves not administered Lactipro. It is unclear why these results differ, but it is important to note that Muya et al. (2015) collected rumen samples $42 \mathrm{~d}$ postinoculation only and that rumen populations of $M$. elsdenii were never determined. This was a main objective here and makes the work of Muya et al. (2015) more correlative than causative. Also, the control calves used herein were fed autoclaved Lactipro, whereas Muya et al. (2015) did not feed control calves autoclaved Lactipro. Ingredients in the probiotic broth may have been utilized by the calf and the rumen microbiota, which could explain lack of agreement between the results reported herein and Muya et al. (2015).

Van Soest (1994) stated that bacteria would establish in the rumen if their growth rate was greater than the passage rate of the rumen. It was expected that the rumen environment would favor M. elsdenii establishment due to the presence of substrate (e.g., sugars and lactate), but the rumen passage rate in young calves is variable (depending on health) and is not well defined. The generation of $M$. elsdenii varies depending on the strain, substrate, and media but probably ranges from 170 to 400 min (Forsberg, 1978). However, the growth rate is probably much lower in the rumen than in the laboratory media, which likely affected $M$. elsdenii establishment in the rumen.

In conclusion, a single oral administration of Lactipro, a commercial M. elsdenii probiotic, to 14-d-old

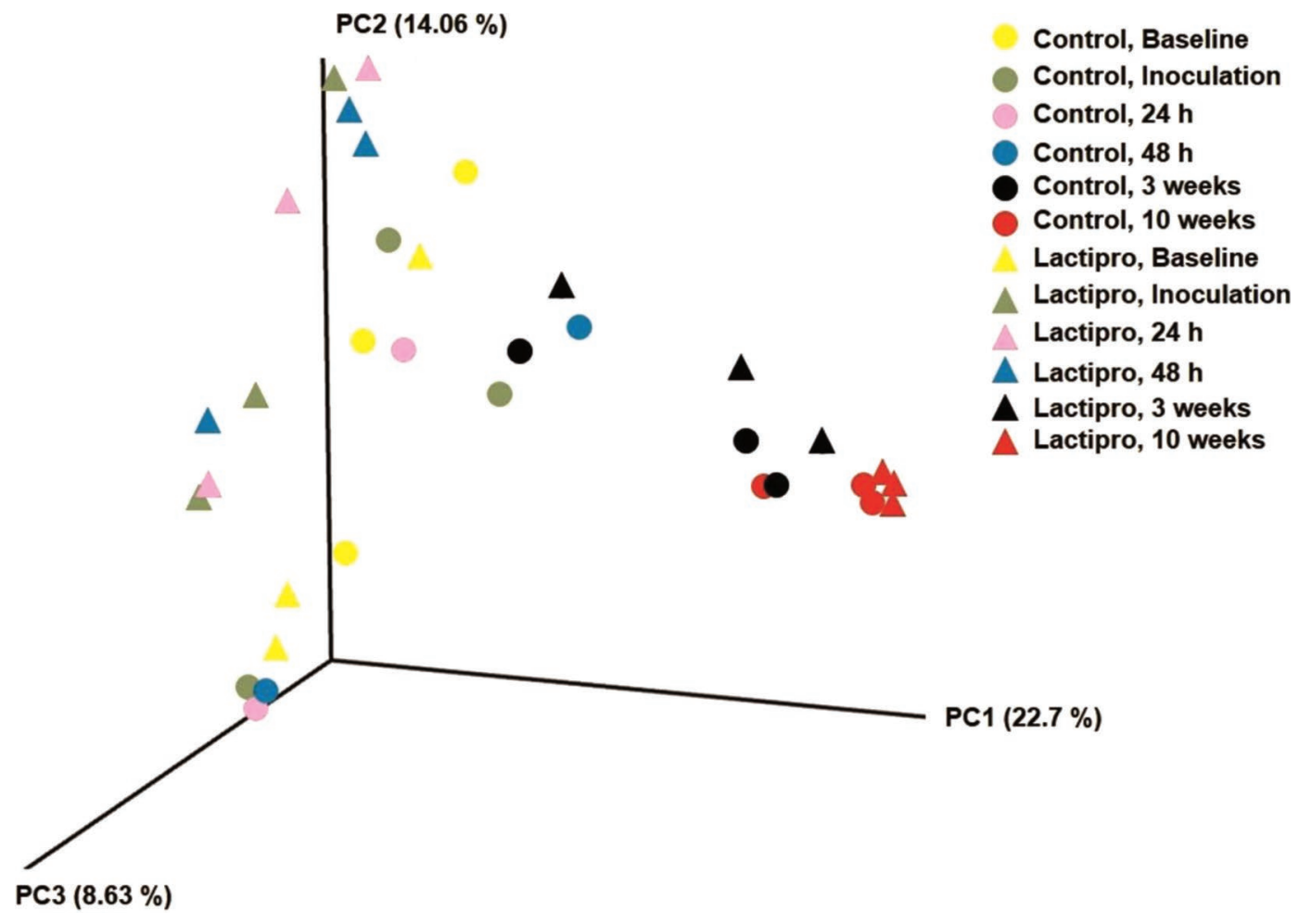

Figure 2. Principal coordinates (PC) analysis of rumen microbiome. Control (gray shapes) calves received $25 \mathrm{~mL}$ of autoclaved Lactipro; Lactipro (black shapes) calves received $25 \mathrm{~mL}$ of nonautoclaved Lactipro, a commercially available probiotic product of Megasphaera elsdenii. Calves were dosed on d 14 of the trial. Within treatment, rumen content samples were obtained: before dosing (baseline), at inoculation, 24 and $48 \mathrm{~h}$ after inoculation, and 3 and $10 \mathrm{wk}$ after inoculation. One sample was removed from control, $24 \mathrm{~h}$ and control, $48 \mathrm{~h}$ during the data filtering process (see text); remaining groups have 3 replicates each. Color version available online. 
calves shifted the population of some minor rumen bacteria, but it did not result in a sustained increase of the rumen Megasphaera population. As a result, neither rumen and blood metabolite concentrations nor calf growth were significantly influenced by Lactipro administration. Multiple dosing may be needed to bring appreciable effects.

\section{ACKNOWLEDGMENTS}

This work was supported by a Virginia Ag Council (Richmond) grant awarded to K. M. Daniels (2015). Product donation from MS Biotec (Wamego, KS) is acknowledged. Connor Owens and Kellie Enger (graduate students at Virginia Tech, Blacksburg) are thanked for their assistance with feeding and collecting samples.

\section{REFERENCES}

Aikman, P. C., P. H. Henning, D. J. Humphries, and C. H. Horn. 2011. Rumen $\mathrm{pH}$ and fermentation characteristics in dairy cows supplemented with Megasphaera elsdenii NCIMB 41225 in early lactation. J. Dairy Sci. 94:2840-2849.

Amaretti, A., E. Tamburini, T. Bernardi, A. Pompei, S. Zanoni, G. Vaccari, D. Matteuzzi, and M. Rossi. 2006. Substrate preference of Bifidobacterium adolescentis MB 239: Compared growth on single and mixed carbohydrates. Appl. Microbiol. Biotechnol. 73:654-662.

Aronesty, E. 2011. ea-utils: Command-line tools for processing biological sequencing data. Accessed Jan. 30, 2015. https://github.com/ ExpressionAnalysis/ea-utils.

Bokulich, N. A., S. Subramanian, J. J. Faith, D. Gevers, J. I. Gordon, R. Knight, D. A. Mills, and J. G. Caporaso. 2013. Quality-filtering vastly improves diversity estimates from Illumina amplicon sequencing. Nat. Methods 10:57-59.

Caporaso, J. G., J. Kuczynski, J. Stombaugh, K. Bittinger, F. D Bushman, E. K. Costello, N. Fierer, A. G. Peña, J. K. Goodrich, J. I. Gordon, G. A. Huttley, S. T. Kelley, D. Knights, J. E. Koenig, R. E. Ley, C. A. Lozupone, D. McDonald, B. D. Muegge, M. Pirrung, J. Reeder, J. R. Sevinsky, P. J. Turnbaugh, W. A. Walters, J. Widmann, T. Yatsunenko, J. Zaneveld, and R. Knight. 2010. QIIME allows analysis of high-throughput community sequencing data. Nat. Methods 7:335-336.

Counotte, G. H. M., R. A. Prins, R. H. A. M. Janssen, and M. J. A. deBie. 1981. Role of Megasphaera elsdenii in the fermentation of dl- $[2-13 \mathrm{C}]$ lactate in the rumen of dairy cattle. Appl. Environ. Microbiol. 42:649-655.

Edgar, R. C. 2010. Search and clustering orders of magnitude faster than BLAST. Bioinformatics 26:2460-2461.

Elsden, S. R., F. M. Gilchrist, D. Lewis, and B. E. Volcani. 1956. Properties of a fatty acid forming organism isolated from the rumen of sheep. J. Bacteriol. 72:681-689.

Forsberg, C. W. 1978. Nutritional characteristics of Megasphaera elsdenii. Can. J. Microbiol. 24:981-985.

Gentile, A., S. Sconza, I. Lorenz, G. Otranto, G. Rademacher, P. Famigli-Beramini, and W. Klee. 2004. D-lactic acidosis in calves as a consequence of experimentally induced ruminal acidosis. J. Vet. Med. A Physiol. Pathol. Clin. Med. 51:64-70.

Haas, B. J., D. Gevers, A. M. Earl, M. Feldgarden, D. V. Ward, G. Giannoukos, D. Ciulla, D. Tabbaa, S. K. Highlander, E. Sodergren, B. Methé, T. Z. DeSantisHuman Microbiome Consortium, J. F. Petrosino, R. Knight, and B. W. Birren. 2011. Chimeric 16S rRNA sequence formation and detection in Sanger and 454-pyrosequenced PCR amplicons. Genome Res. 21:494-504.
Henning, P. H., C. H. Horn, K.-J. Leeuw, H. H. Meissner, and F. M. Hagg. 2010. Effect of ruminal administration of the lactate-utilizing strain Megasphaera elsdenii ( $M e$ ) NCIMB 41125 on abrupt or gradual transition from forage to concentrate diets. Anim. Feed Sci. Technol. 157:20-29.

Hobson, P. N., S. O. Mann, and A. E. Oxford. 1958. Some studies on the occurrence and properties of a large gram-negative coccus from the rumen. J. Gen. Microbiol. 19:462-472.

Kigerl, K. A., J. C. Hall, L. Wang, X. Mo, Z. Yu, and P. G. Popovich. 2016. Gut dysbiosis impairs recovery after spinal cord injury. J. Exp. Med. 213:2603-2620.

Kim, Y.-H., R. Nagata, N. Ohtani, T. Ichijo, K. Ikuta, and S. Sato. 2016. Effects of dietary forage and calf starter diet on ruminal $\mathrm{pH}$ and bacteria in Holstein calves during weaning transition. Front. Microbiol. 7:1575.

Klieve, A. V., D. Hennessy, D. Ouwerkerk, R. J. Forster, R. I. Mackie, and G. T. Attwood. 2003. Establishing populations of Megasphaera elsdenii YE 34 and Butyrivibrio fibrosolvens YE 44 in the rumen of cattle fed high grain diets. J. Appl. Microbiol. 95:621-630.

Klotz, J. L., and R. N. Heitmann. 2006. Effects of weaning and ionophore supplementation on selected blood metabolites and growth in dairy calves. J. Dairy Sci. 89:3587-3598.

Kristensen, N. B. 2000. Quantification of whole blood short-chain fatty acids by gas chromatographic determination of plasma 2-chlorethyl derivatives and correction for dilution space in erthyrocytes. Acta. Agric. Scand. 50:231-236.

Mentschel, J., R. Leiser, C. Mülling, C. Pfarrer, and R. Claus. 2001 Butyric acid stimulates rumen mucosa development in the calf mainly by a reduction of apoptosis. Arch. Tierernahr. 55:85-102.

Mikx, F. H. M., and J. S. van der Hoeven. 1975. Symbiosis of Streptococcus mutans and Veillonella alcalescens in mixed continuous cultures. Arch. Oral Biol. 20:407-410.

Muya, M. C., L. J. Erasmus, K. Miller, C. Aperce, F. V. Nherera, and P. M. Moshidi. 2017. Performance of Holstein calves having free access to milk and dosed with Megasphaera elsdenii. Sci. Agric. 74:189-194.

Muya, M. C., F. V. Nherera, K. A. Miller, C. C. Aperce, P. M. Moshidi, and L. J. Erasmus. 2015. Effect of Megasphaera elsdenii NCIMB 41125 dosing on rumen development, volatile fatty acid production and blood betahydroxybutyrate in neonatal dairy calves. J. Anim. Physiol. Anim. Nutr. (Berl.) 99:913-918.

Ng, S. K. C., and I. R. Hamilton. 1971. Lactate metabolism by Veillonella parvula. J. Bacteriol. 105:999-1005.

Quigley, J. D., III, J. K. Bernard, T. L. Tyberendt, and K. R. Martin. 1994. Intake, growth, and selected blood parameters in calves fed calf starter via bucket or bottle. J. Dairy Sci. 77:354-357.

Sander, E. G., R. G. Warner, H. N. Harrison, and J. K. Loosli. 1959. The stimulatory effect of sodium butyrate and sodium propionate on the development of rumen mucosa in the young calf. J. Dairy Sci. 42:1600-1605.

Stevenson, D. M., and P. J. Weimer. 2007. Dominance of Prevotella and low abundance of classical ruminal bacterial species in the bovine rumen revealed by relative quantification real-time PCR. Appl. Microbiol. Biotechnol. 75:165-174.

Trovatelli, L. D., and D. Matteuzzi. 1976. Presence of bifidobacteria in the rumen of calves fed different rations. Appl. Environ. Microbiol. $32: 470-473$.

Van Soest, P. J. 1994. Nutritional Ecology of the Ruminant. 2nd ed. Cornell University Press, Ithaca, NY.

Weimer, P. J., L. Da Silva Cabral, and F. Cacite. 2015. Effects of ruminal dosing of Holstein cows with Megasphaera elsdenii on milk fat production, ruminal chemistry, and bacterial strain persistence. J. Dairy Sci. 98:8078-8092.

Yohe, T. T., K. M. O'Diam, and K. M. Daniels. 2015. Growth, ruminal measurements, and health characteristics of Holstein bull calves fed an Aspergillus oryzae fermentation extract. J. Dairy Sci. 98:6163-6175.

$\mathrm{Yu}, \mathrm{Z}$., and M. Morrison. 2004. Improved extraction of PCR-quality community DNA from digesta and fecal samples. Biotechniques $36: 808-812$. 